- by the correction of azotemia: «nephrostomy > stenting > catheterization»;

- by the correction of urinary syndrome: «catheterization $>$ stenting $=$ nephrostomy»;

- by the correction of PCS-dilatation: «nephrostomy > stenting > catheterization»;

- by the general clinical effectiveness: «stenting = nephrostomy > catheterization»;

- in terms of overall effectiveness: «nephrostomy > stenting $>$ catheterization».

Conclusion. Based on the results obtained, it should be concluded that the effectiveness of the compared methods of drainage of the upper urinary tract (internal stenting of the ureter, catheterization of the ureter, percutaneous puncture nephrostomy) in most cases has a difference both in particular and in complex indicators. Nevertheless, when choosing specific drainage methods, it is necessary to move from taking into account their individual advantages and disadvantages to developing a unified selection system. In the process of choosing a method as backbone factors, one should focus on the actual indicators of a specific clinical situation that are important for drainage. To increase the efficiency of the choice of the method of drainage of the upper urinary tract, its integral assessment is necessary, which is proved by the example of the results of this study. The results obtained should be taken into account in urological practice.

$$
* * *
$$

1. Fedorov A.A. Comparative assessment of azotemia indices after different variants of upper urinary tract drainage / A.A. Fedorov, O. V. Zolotukhin, Yu.Yu. Madykin, A.V. Petryaev, S.N. Titov, V.O. Zolotukhin // Experimental and Clinical Urology. - 2019. - № 4. - P. 92-99. - ISSN 2222-8543, DOI: 10.29188 / 2222-8543-2019-11-4-92-99, E-library ID: 41578502. - Text (visual): immediate + Text (visual): electronic.

2. Zolotukhin O.V. Evolution of the method of drainage of the upper urinary tract / O.V. Zolotukhin, Yu.Yu. Madykin, A.A. Fedorov // Bulletin of experimental and clinical surgery. - 2019. - T. 12, № 3. P. 199-202. - ISSN: 2070-478X, eISSN: 2409-143X, DOI: 10.18499 / 2070-478X-2019-12-3-199-202, E-library ID: 39197642. - Text (visual): immediate + Text (visual): electronic.

3. Fedorov A.A. Certificate of state registration of the database № 2020621687, 19.09.2020. «Upper urological obstruction data base - 1.0 (UUO-DB 1.0)»/ A.A. Fedorov, O. V. Zolotukhin, Yu.Yu. Madykin, V.O. Zolotukhin, P.A. Popov // Federal Institute of Industrial Property (Rospatent website) [electronic resource]. $\quad-\quad$ https://new.fips.ru/registers-docview/fips_servlet?DB=DB\&rn=5878\&DocNumber=2020621687\&TypeFile=html (date of application: 11.13.2020). - Text (visual): electronic.

4. Fedorov A.A. Certificate of state registration of a computer program № 2020611592, 02.04.2020. «Upper urological obstruction - expert 1.0 (UUO-expert 1.0)» / A.A. Fedorov, O. V. Zolotukhin, Yu.Yu. Madykin, V.O. Zolotukhin, P.A. Popov, E.A. Baranov // Federal Institute of Industrial Property (Rospatent website) [electronic resource]. - $\quad$ https://new.fips.ru/registers-docview/fips_servlet?DB=EVM\&rn=1515\&DocNumber=2020611592\&TypeFile=pdf (date of application: 11.13.2020). - Text (visual): electronic.

5. Bakanov M.I. The theory of economic analysis / M.I. Bakanov, A.D. Sheremet. - Moscow, 2001. - 416 p. - Text (visual): electronic.

\title{
Moskalets O.V. \\ Anti-rituximab antibodies in refractory / relapsing cases of chronic lymphocytic leukemia
}

Moscow Regional Research Clinical Institute named after M.F.Vladimirskij

(Russia, Moscow)

doi 10.18411/gq-30-11-2020-02

idsp sciencerussia-30-11-2020-02

\section{Abstract}

Rituximab, a chimeric monoclonal antibody targeted against the pan-B-cell marker $\mathrm{CD} 20$, is widley used for treatment of lymphomas, rheumatologic diseases and other 
disorders. It is known that many monoclonal antibodies such as rituximab can elicit anti-drug antibodies, which may interfere with therapeutic response. The aim of this study was to investigate the incidence of antibodies to rituximab in patients with chronic lymphocytic leukemia. Serum concentrations of anti-rituximab antibodies was determined in blood serum of patients with B-chronic lymphocytic leukemia (newly diagnosed and resistant / recurrent forms, previously treated by rituximab) and healthy controls. Results: none of the patients with newly diagnosed disease have antibodies to rituximab. Positive results were recorded in $8(33 \%)$ patients who received rituximab earlier.

Key words: rituximab, anti-drug antibody, chronic lymphocytic leukemia.

Introduction Currently, genetically engineered drugs are widely used to treat hemoblastosis and autoimmune diseases [1,2]. Because of their higher target specificity, monoclonal antibodies treatments are generally considered to pose a lower risk of adverse reactions than chemical drugs. Rituximab, a chimeric monoclonal antibody targeted against the pan-B-cell marker CD20, was the first monoclonal antibody to be approved for therapeutic use [2]. It is usually used in combination with chemotherapy. Treatment with rituximab at standard weekly dosing is effective in more than $50 \%$ of patients with relapsed or refractory CD20-positive follicular non-Hodgkin's lymphoma, but is not curative. It is less effective in other subtypes of CD20-positive lymphoma and for retreatment, even with CD20 still expressed [3]. Thus, binding of rituximab to CD20 is not sufficient to kill many lymphoma cells, indicating that there are mechanisms of resistance. Mechanisms of cell destruction that have been demonstrated to be activated by rituximab binding to CD20 include direct signaling of apoptosis, complement activation and cell-mediated cytotoxicity [1]. The relative importance of each of these mechanisms in determining clinical response to rituximab treatment remains a matter of conjecture. Thus, the role of various resistance pathways, some documented in experimental systems and others still hypothetical, remains uncertain. Resistance could potentially be mediated by alterations in CD20 expression or signaling, elevated apoptotic threshold, modulation of complement activity or diminished cellular cytotoxicity [1]. Besides, immunogenicity, in particular the induction of anti-drug antibodies (ADAs), is an important concern, and thus immunogenicity assessment is a requirement for their approval. [4] The literature actively discusses the issue of the lack of efficacy of rituximab in some patients due to ADAs [5-7].

The aim of this pilot study was to investigate the incidence of antibodies to rituximab in patients with chronic lymphocytic leukemia.

Matherials and methods. The study involved 36 patients with B-chronic lymphocytic leukemia (B-CLL) (12 newly diagnosed, 24 - resistant / recurrent form), aged from 35 to 68 years (average age 49,7 years), of witch 21 women and 15 men and and 12 practically healthy individuals, matched by age and sex. In $60 \%$ of patients, stage 2 of the disease was detected, in $40 \%$ of patients - stages 3 and 4 . Patients with refractory / relapsing forms have previously received 4-6 courses of R-FC therapy. Determination of antibodies to rituximab in peripheral blood serum was performed by enzyme-linked immunoassay using a test system manufactured by Bender Medsystems (Austria) (semi-quantitative analysis)..A typical hemolytic uremic syndrome is a disease from the group of thrombotic microangiopathies, the cause of which is an infection caused by Shiga toxin (Stx) -producing microorganisms, primarily enterohemorrhagic Escherichia coli. The disease most often affects children from 6 months to 5 years, although it is possible at any age, manifested by the development of a triad of symptoms - hemolytic anemia, thrombocytopenia, acute renal damage - after the prodromal period of acute intestinal infection, often hemocolitis. [1,2,3]

Results None of the patients with newly diagnosed disease have antibodies to rituximab. Positive results were recorded in $8(33 \%)$ patients who received rituximab earlier. Of particular note is a patient with a very high antibody level (74,7 c.u.) who received 6 courses of combination therapy and showed pronounced progression of the disease. At the 
same, in the group of practically healthy individuals, positive results were obtained in 2 cases, in particuilar, with a procedural nurse who regularly contacts the drug.

Conclusion. Anti-rituximab antibodies were detected in every third patient with an unfavorable course of the disease. If we suppose that immunogenicity is an important factor that should be considered in the overall treatment strategy, we should take actions to reduce antidrug antibodies formation: modifying drug administration; increasing dose; decreasing immunogenicity by adding immunosuppressive agents to the regimen or using new drugs which are supposed to be less immunogenic such as humanized or fully human monoclonal antibodies.

1. Chan A.C., Carter R.T. Therapeutic antibodies for autoimmunity and inflammation. Nat. Rev. Immunol. 2009. Vol.10. P.301-316. Doi:10.1038/nri2761/

2. Smith M.R. Rituximab (monoclonal anti-CD20 antibody): mechanisms of actionand resistance. Oncogene. 2003. Vol.22(47). p.7359-7368. Doi 10.1038/sjonc120639.

3. Maloney D.R. Anti-CD20 antibody therapy for B-cell lymphomas. N Engl. J. Med. 2012.Vol.316. P.2008-2018 DoI:10/1056/NEJMct1114348.

4. Rosenberg A.S., Sauna Z.E.Immunogenecity assesment during the development of protein therapeutics. J. Pharm. Pharmacol. 2017. Doi:10.1111/jphp12810.

5. Krishna M., Nadler S.G. Immunogenecity to biotherapeutics - the role of anti-drug immune complexes. Front. Immunol. 2016; 7:21 Doi: 10.3389/fimmu.2016.00021.

6. Tada M., Ishii-Watabe A., Suzuki T., Kawasaki N. Development and characterisationof an anti-rituximab monoclonal antibodies. Mabs. 201 ' 8. Vol.10(3). P.370-379. Doi: 10.1080/19420862.2018.1424610.

7. vanSchi K.A., Wolbink G.J., Respens T. Cross-reactive and pre-existing antibodiesto therapeuticantibodies - Effects on treatmentand immunogenecity. Mabs. 2015. Vol.7. 662-671. DoI:10.1080/19420862.2015.10481111.

Tsukanova E.A., Sushchenko A.V., Podoprigora A.V., Popov P.A. The interaction of methylacrylic polymer materials of removable plate prosthetics and the mucous membrane of the prosthetic bed of patients with partial secondary adentia and concomitant gastrointestinal diseases: a comparative analysis of complications

Voronezh State Medical University named after N.N. Burdenko of the Ministry of Health of the Russian Federation

(Russia, Voronezh)

doi 10.18411/gq-30-11-2020-03

idsp sciencerussia-30-11-2020-03

\section{Abstract}

The article presents the data of an original study on the improvement of orthopedic treatment of partial secondary adentia in patients with concomitant chronic pathology of the gastrointestinal tract by the method of removable plate prosthetics. For this purpose, a comparative analysis of three alternative to each other preparations of methyl acrylic polymer for the manufacture of bases of prostheses was made: 1) «GC», 2) «3M-Espe», 3) «Ftorax». A scientific assumption has been formulated that the choice of a specific polymer preparation (within the comparison base) will lead to a change in the number of complications developing during the operation of the prosthesis. In three independent groups of patients, using the compared preparations of methyl acrylic polymer, the relative number of complications was estimated. After the implementation of the study, it was found that the most positive results are shown by the polymer «GC», the least positive - by the polymer «Ftorax», the polymer «3M-Espe» occupies an «intermediate position». The results obtained have a high level of statistical significance, which makes it possible to recommend them for consideration for use in the practice of orthopedic dentistry.

Key words: partial secondary adentia, chronic pathology of the gastrointestinal tract, removable plate prosthetics, methyl acrylic polymers, complications. 